\title{
MODELO HÍBRIDO: GESTIÓN DEL CAMBIO E INNOVACIÓN SOCIAL
}

\author{
Sidia B. Moreno R. ${ }^{\text {; }}$ \\ Elba J. de Carrizo 2;
}

\begin{abstract}
:
Research consisted in contrasting two approaches used in the development of technology-based social and organizational transformation projects. One from the social sphere and one from the business sector. This with the purpose of generating the basis for a new hybrid model that incorporates aspects of both approaches, in order to strengthen processes of co-creation, co-execution and management of organizational change in crisis environments. To this end, a qualitative evaluation was carried out on the phases of the Systemic Methodology of Change Management used in the business sector, comparing it with the phases and criteria utilized in the projects of the Social Innovation Lab of the Technological University of Panama. A comprehensive assessment of the methodology was obtained, in accordance with the social innovation approach, and a set of desirable characteristics in the hybrid model.
\end{abstract}

Keywords: social transformation, organizational change, co-creation, co-execution

\section{Resumen:}

La investigación consistió en la contrastación de dos enfoques utilizados en el desarrollo de proyectos de transformación social y organizacional de base tecnológica. Uno del ámbito social y el otro empresarial, para generar la fundamentación de un nuevo modelo híbrido que incorpore aspectos de ambos enfoques que fortalezcan procesos de cocreación, coejecución y de gestión del cambio organizacional en ambientes de crisis. Para ello, se realizó una evaluación cualitativa de las fases de la Metodología Sistémica e Integral del Cambio utilizada en el sector empresarial, y se comparó con las fases y criterios de los proyectos del Labotarorio de Innovación Social de la Universidad Tecnológica de Panamá. Se obtuvo la valoración integral de la metodología, en función del enfoque de innovación social y se identificaron características deseables en el modelo híbrido a proponer. coejecución

Palabras-claves: transformación social, cambio organizacional, cocreación,

${ }^{1}$ CINEMI - Universidad Tecnológica de Panamá (UTP), Panamá. Correo electrónico: sidia.moreno@utp.ac.pa

2 Dirección de Desarrollo Organizacional - Inversiones Bahía Ltd., Panamá. Correo electrónico: elbacarrizo66@gmail.com

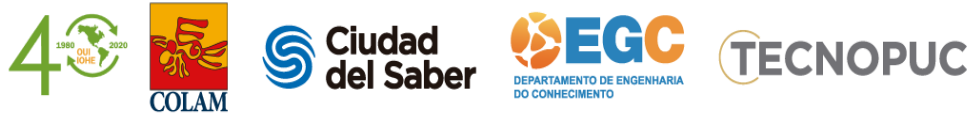




\section{FUNDAMENTACIÓN}

\section{1 CARACTERÍSTICAS DEL CAMBIO SOCIAL}

Señala Briceño (2011) que cada vez más se hace necesaria una nueva visión orientada por enfoques preventivos y adaptativos para administrar racionalmente los sistemas complejos de transformación, donde se pueden incluir los sistemas naturales y humanos que interactúan sistémicamente, para propiciar sustentabilidad, lo cual depende esencialmente de comprender los problemas y restricciones de la dinámica evolucionista. Señala, además que, en determinadas circunstancias, cuando los sistemas se encuentran sobre tensionados, los procesos de cambio no son graduales, sino que surgen bifurcaciones y saltos bruscos, que generan una evolución cultural acelerada, en la que las personas se transforman porque tienen que hacerlo y porque poseen capacidad de analizar, planificar, gestionar y alterar el curso de su desarrollo y calidad de vida. Tal es el caso de las repercusiones a nivel mundial, provocadas por la COVID19, en la que las organizaciones y personas están tratando de adaptarse a condiciones repentinas como el teletrabajo y la fuerte inestabilidad financiera que indudablemente irrumpen, tanto en las organizaciones, como en el núcleo familiar y, por ende, en el desarrollo de las comunidades.

Escalante y Miñano (1982), por su parte, identifican el cambio social con la noción de progreso, entendida como evolución sociocultural. Ellos hacen referencia a las variaciones innovativas efectuadas en el marco de una cultura. Se trata de un mecanismo activo para el mejoramiento social que afecta su estructura y funcionamiento a merced de la variedad y frecuencia de intercambios que se establecen entre los elementos de la cultura con otros de carácter foráneo, lo que se conoce como difusión. El concepto de innovación, lo utilizan para referirse a un nuevo elemento que se pone a disposición de la cultura. En este sentido, Roger (2003), en su teoría de la Difusión de Innovación, hace una conceptualización aplicable al cambio social y proceso de gestión del cambio que prioriza la dimensión humana por encima de los procesos y las herramientas tecnológicas; en el que el proceso de transformación tiene lugar en un sistema complejo y adaptativo y cuando la evolución cultural se acelera a causa de situaciones críticas e inesperadas. Se generan, entonces, nuevas condiciones que podrían

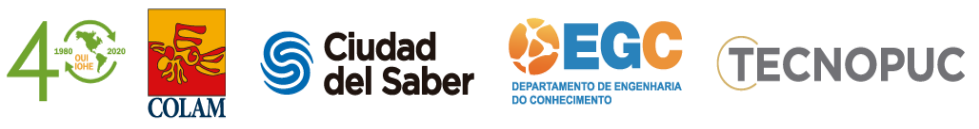


propiciar progreso o mejoramiento social, siempre y cuando haya variaciones innovativas en las estructuras y funcionamiento en el lugar en el que se desenvuelven las personas.

\subsection{GESTIÓN DEL CAMBIO, HABILITADOR DEL DESARROLLO HUMANO}

En la gestión del cambio organizacional, es vital considerar los aspectos que inciden en el ser humano y su disposición para esto. Los miedos naturales que llevan a una resistencia racional o totalmente irracional que generan altos niveles de estrés individual, deben ser atendidos desde las etapas iniciales de la situación que se enfrenta (Vakola y Nikolaou, 2005). Identificar aspectos culturales de las organizaciones en las que se desempeñan las personas, o bien el sistema de creencias de una comunidad, su historia y valores, son fundamentales para acompañar el desafío de cambio; sea éste la implementación de nuevas tecnologías, una restructuración por fusión o adquisición $(F \& A)$, o más profundamente, la adaptación de nuevas formas de operar en un entorno comunitario frente a la necesidad de solucionar problemas.

Las investigaciones sobre transformaciones organizacionales basadas en la Encuesta Global de McKinsey (2010), confirman tendencias de cambio que se han mantenido en el tiempo. Aún cuando pocos son los ejecutivos que dicen haber trabajado en transformaciones exitosas (entre 20\% 26\%), queda en evidencia que las empresas que lograron resultados significativos implementaron un conjunto de 24 acciones, en su mayoría relacionadas a factores humanos. Al respecto Roger (2007) plantea que la adopción de una innovación tiene que ver con su conocimiento, persuasión y decisión, todos aspectos del comportamiento humano.

\subsection{GESTIÓN DEL CAMBIO ORGANIZACIONAL}

El cambio está impulsado por factores del entorno global donde opera la organización, tales como: las fuerzas del mercado, los avances tecnológicos, los competidores, nuevas regulaciones, o bien las grandes crisis como la pandemia de la COVID-19. Estos factores, al igual que otros internos, alteran el modelo operativo de una empresa. Los factores del entorno son catalogados como turbulentos y complejos, por lo que ameritan un abordaje sistémico. Por

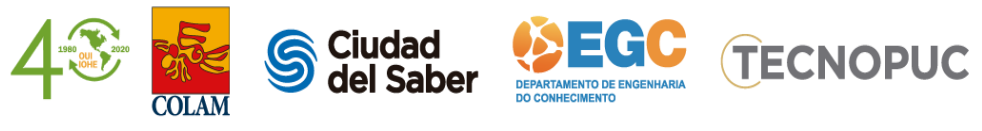


ejemplo, en la época de los 80 y 90, grandes multinacionales incursionaron en una aproximación estructurada y sistémica de sus procesos de cambio, impulsados por la tendencia de Fusión y Adquisición $(F \& A)$; surgiendo el modelo de la General Electric, Change Acceleration Process (CAP), inspirado en la teoría de Kotter (1996). Con el tiempo se fueron adaptando los procesos de cambio a las particularidades de sus organizaciones y como resultado van surgiendo nuevas metodologías, como la de Alineación Sistémica del Cambio, generada por HENKA (2013), que contempla cinco fases del proceso de cambio, influenciadas por las fuerzas a favor y en contra (Lewin, 2018). Estas fases son las siguientes: Sensibilización del Cambio; Análisis y Comprensión del Cambio; Alineación Organizacional; Despliegue del Cambio y; Soporte y Afianzamiento.

Todnem (2005), sostiene en su enfoque de Cambio Emergente, que el cambio organizacional debe ser gestionado desde la complejidad que surge de las interacciones, interdependencias y coevoluciones que se generan en las distintas partes del "ecosistema" o entorno (estrategia, procesos, personas, liderazgo, tecnología, estructura organizacional, cultura), lo cual se sustenta desde la Teoría de la Complejidad (Mitleton-Kelly, 2003), utilizada para generar una nueva metodología aplicada empresarialmente y que servirá para diseñar el modelo híbrido a proponer. Las lecciones aprendidas durante el desarrollo y análisis de procesos de gestión del cambio desde hace más de10 años, en un número significativo de casos en Panamá y otros países de América Latina, complementadas con componentes del modelo $C A P$ y la Metodología HENKA, dan como resultado la Metodología de Gestión Integral del Cambio (MGSIC), que considera, a su vez, la Teoría del Cambio por Etapas (Lewin, 2018), a saber: i) consciencia del problema percibido por el liderazgo, los equipos de trabajo y los contribuidores individuales; ii) la decisión consciente de adoptar una innovación (sé/no sé y quiero adoptar lo nuevo); iii) la implementación que incluye la modificación de las estructuras organizacionales para alinearlas; y iv) la institucionalización o asimilación de la innovación en la organización. La metodología adiciona nuevos elementos, como lo son, herramientas transversales de acompañamiento de Coaching Ejecutivo y Coaching de Equipos, así como un Marco de Control Interno (MCI) para la gestión de los procedimientos y políticas que hacen parte del modelo operativo de la empresa. Además, la MGSIC, incorpora dos técnicas para la generación y diseño de ideas innovadoras: Art of Hosting y Design Thinking (Turner y Croy, 2010). Sus etapas son

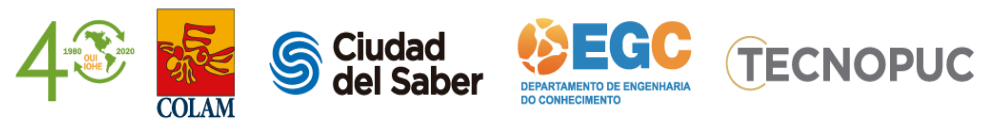


las siguientes: Sensibilización; Diseño y Desarrollo; Alineación Organizacional; Ejecución y Sostenibilidad. Apoyadas por acciones transversales de comunicación, coaching y capacitación.

\subsection{INNOVACIÓN SOCIAL Y GESTIÓN DEL CAMBIO}

El trabajo conjunto de las universidades, las empresas y los gobiernos en términos de contribución al desarrollo de los países, puede aportar de manera significativa a la gestión del cambio en comunidades o territorios, teniendo en cuenta que las organizaciones están conformadas por personas de una sociedad. Desde esta perspectiva, se desarrolla la presente investigación, producto del trabajo conjunto entre el Centro de Investigación e Innovación Eléctrica, Mecánica y de la Industria (CINEMI) de la Universidad Tecnológica de Panamá y sectores empresariales con experiencia en temas de gestión de innovación tecnológica.

La innovación social incorpora criterios fundamentales que tienen que ver con la generación colectiva de nuevas ideas (cocreación) para solucionar problemas o aprovechar nuevas oportunidades, mediante la participación activa de los afectados; desde la generación de las ideas, hasta el desarrollo de las soluciones (coejecución); teniéndose en cuenta criterios de transformación social, repetitividad y sostenibilidad de los resultados (Moreno, S. y otros, 2019); para lo cual se requieren actitudes y aptitudes en las personas afectadas. Esto puede tomar la forma de generación de nuevos y mejores productos, servicios, o mejoras de procesos o; modelos organizativos participativos y novedosos para solventar necesidades básicas; o simplemente nuevos canales para acceder de manera efectiva y equitativa a los bienes públicos. Los modelos y metodologías que se aplican para este propósito guardan relación con las nuevas metodologías de gestión del cambio organizacional que se utilizan en el sector empresarial, sobre todo, en lo relacionado con el abordaje de la dimensión humana y la transformación social.

El enfoque de innovación social en los proyectos desarrollados por el Laboratorio de LabIS-UTP (Moreno y otros, 2019), considera tres procesos claves: Sensibilización, Cocreación y Coejecución, alimentados y fortalecidos por un proceso transversal de Feedback que se mantiene durante todo el ciclo de vida de sus proyectos. La Sensibilización tiene por

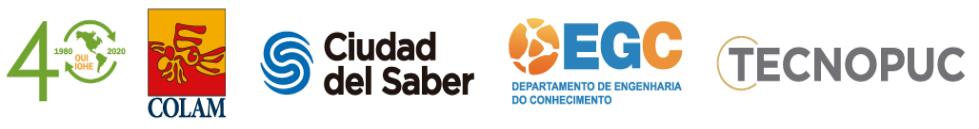


objetivo identificar y socializar de manera colectiva elementos que le hagan sentido a los integrantes de la comunidad demandante, actores y colaboradores. La Cocreación, contempla cuatro momentos en el proceso: i) inducción, ii) innovación, iii) validación y iv) propuesta integral. La Coejecución constituye la implementación de las soluciones de manera colectiva. Los aspectos metodológicos del enfoque están basados en la técnica de análisis policontextural (Briceño, 2011) y en los criterios RENGIS (Tabla 1).

\section{METODOLOGÍA DE LA INVESTIGACIÓN}

Se realizó un análisis comparativo del enfoque de Innovación Social del LabIS-UTP y sus respectivos criterios de IS (Tabla 1), con las fases de la MGSIC. Se seleccionaron dos experiencias empresariales de gestión de cambio (Caso 1 y Caso 2), teniendo en cuenta para la selección: el cambio o transformación de personas o grupos de personas; la tecnología como dinamizador del cambio a producir, y procesos con enfoque sistémico y sistemático. La descripción de los casos (Tabla 2), se hizo según las siguientes preguntas de evaluación: ¿qué cambios se requerían implementar y con qué estructura y recursos se contaba?; ¿cómo se abordó la dimensión humana del cambio para generar compromiso en el proyecto?; ¿cómo se garantizó el éxito del cambio?; ¿cómo se midió el éxito del cambio? Y ¿cómo se gestiona la sostenibilidad del cambio? Las respuestas a estas preguntas se presentan en las Tablas 3 y 4. La identificación de las coincidencias en los enfoques y su respectiva valoración, se describen en la Tabla 5. Se utilizó la siguiente escala descriptiva: BAJO- coincide en 35\% o menos; MEDIO - coincide entre el 36\% - 69\%; ALTO - coincide entre el 70\%-100\%. Finalmente, se resumieron los resultados según el nivel de coincidencia o presencia en la Tabla 6.

\section{DATOS DE LA INVESTIGACIÓN}

\subsection{DESCRIPCIÓN DE LOS CASOS}

Los datos fueron generados de dos empresas multilatinas con base en Panamá, con distintos desafíos de negocios de crecimiento y de regionalización.

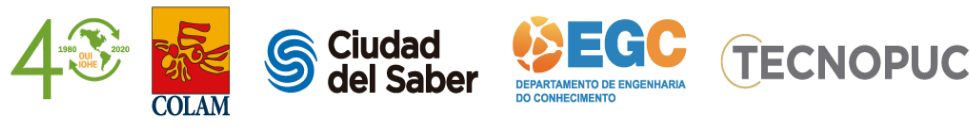


Tabla 1: Criterios para la Evaluación de Proyectos de Innovación Social del LabIS-UTP

\section{Criterios/Indicadores 1, 2, 3 y 4 \\ C1: SOSTENIBILIDAD}

1.1 Se propicia el liderazgo comunitario de manera efectiva.

1.2 Se cuenta con los recursos necesarios para su funcionamiento

1.3 Se cuenta con mecanismos de autogestión

1.4 Es posible funcionar sin que haya aportes externos

1.5 Se cuenta con mecanismos de autoevaluación y retroalimentación

1.6 Se propicia el aprendizaje continuo en la comunidad

1.7 El aprendizaje fomenta nuevos liderazgos

1.8 Se fomenta la participación intercultural e intersectorial

\section{2: IMPACTO SOCIAL}

2.1 La cantidad de población beneficiada (por género, edad, educación y origen) es significativa

2.2 El impacto en la calidad de vida de la población beneficiada (oportunidad de empleo, educación, tenencia de tierra, salud, mejora del ambiente, disponibilidad agua 6.3 potable, entre otros) es significativo
Criterios/Indicadores 5, 6, 7 y 8

C5: INICIATIVA GENUINA

5.1 El proyecto atiende a una necesidad no atendida e identificada colectivamente por la comunidad

5.2 Las soluciones a los problemas se identifican, diseñan y ejecutan de manera colectiva en la comunidad

5.3 Existe representatividad en la participación para identificar problemas y generar soluciones.

5.4 El proyecto es acogido de manera efectiva por la mayoría de la comunidad

5.5 Nace el proyecto por iniciativa de la comunidad

5.6 Se utilizaron estrategias para la participación ciudadana

\section{C6: INICIATIVA CONSOLIDADA}

6.1 El proyecto se encuentra en fase experimental

6.2 Existen experiencias documentadas sobre prácticas realizadas para validar el grado de atención a las necesidades y oportunidades identificadas

6.3 Se cuenta con una estrategia de mitigación de riesgos

6.4 Se ha desarrollado o desarrolla un prototipo de solución o proyecto piloto

6.5 El diseño de la solución ha necesitado ser modificado a causa de la revisión de requerimientos genuinos

\section{3: CAPACIDAD TRANSFORMADORA}

3.1 La cantidad de población beneficiada (por género, edad, educación y origen) es significativa

3.2 Se cumple los indicadores de resultados del proyecto.

3.3 Se contribuye al cambio de conductas y hábitos en la comunidad

3.4 Se fomenta lar participación con todos los actores

3.5 Se generan nuevos compromisos colectivos para mejorar la comunidad

3.6 Se generan o propician nuevas estrategias para abordar conflictos y situaciones adversas

3.7 Se poseen mecanismos permanentes de retroalimentación

3.8 Se articula el proyecto con nuevos procesos en la comunidad

3.9 Se genera cohesión social con el proyecto

C4: ORIGINALIDAD:

4.1 La propuesta de solución constituye un cambio de paradigma

4.2 Se trata de una experiencia única o diferente de lo actuado anteriormente

4.3 Se aplica en la solución las mejores prácticas de gestión social y tecnológica existentes en el ámbito abordado

\section{C7: REPETITIVIDAD DE LA SOLUCIÓN}

7.1 Deja el proyecto documentación sobre experiencias y lecciones aprendidas que permite ser aplicado a otros escenarios

7.2 Se Fomenta la generación de informes y guías que incorporen las lecciones aprendidas

7.3 Se tiene la posibilidad de que el proyecto se convertirse 0 contribuya a una práctica permanente 0 aporte a una política pública 0 en referente para normativos 0 nuevos programas sociales nacionales y regionales

7.4 Se documentan lecciones aprendidas que permitan preservar las experiencias adquiridas en el proyecto

7.5 Se desarrollan guías y protocolos para la aplicación de las etapas del proyecto

C8: DESAFIO O NECESIDAD VIGENTE

8.1 El problema es una necesidad sentida, priorizada y presente en la comunidad

8.2 Hay correspondencia del problema planteado colectivamente con las propuestas de soluciones

8.3 El proyecto responde a necesidades actuales y sentidas (significativas) de la población meta 
Tabla 2 - Descripción de los casos analizados

\begin{tabular}{|c|c|c|}
\hline Aspectos & Caso 1 & Caso 2 \\
\hline $\begin{array}{l}\text { El desafío } \\
\text { organizacional }\end{array}$ & $\begin{array}{l}\text { Crecimiento y expansión de la empresa } \\
\text { en la Región de Latinoamérica, } \\
\text { principalmente en dos de sus mercados } \\
\text { más grandes, donde se tenía poco o } \\
\text { ninguna experiencia }\end{array}$ & $\begin{array}{l}\text { Consolidar posicionamiento como líder de la } \\
\text { industria en la región Centro América, } \\
\text { mediante la implementación de un modelo } \\
\text { operativo único enfocado en los procesos, la } \\
\text { estructura organizacional y las formas de } \\
\text { operar (nueva cultura organizacional). }\end{array}$ \\
\hline $\begin{array}{l}\text { El impulsor del } \\
\text { cambio }\end{array}$ & $\begin{array}{l}\text { Estrategia de crecimiento, con el } \\
\text { habilitador de la herramienta } \\
\text { tecnológica (ERP). }\end{array}$ & $\begin{array}{l}\text { Estrategia de optimización y control operativo } \\
\text { para asegurar el retorno sobre la inversión en la } \\
\text { compra de la empresa competidora }\end{array}$ \\
\hline Oportunidad & $\begin{array}{l}\text { Aprovechar el esfuerzo y la inversión } \\
\text { con la entrada a nuevos países y el } \\
\text { crecimiento en regiones de países } \\
\text { donde ya se tenía presencia, a fin de } \\
\text { estandarizar el modelo operativo. }\end{array}$ & $\begin{array}{l}\text { Mantener las herramientas tecnológicas de las } \\
\text { distintas operaciones adquiridas en los nuevos } \\
\text { mercados, e ir generando eficiencias en la } \\
\text { gestión a través de la implementación gradual } \\
\text { del nuevo modelo operativo. }\end{array}$ \\
\hline El Alcance & $\begin{array}{l}\text { Operaciones en países existentes, } \\
\text { nuevos países y la Oficina Corporativa, } \\
\text { los cuales operaban bajo un modelo } \\
\text { descentralizado, sin estandarización } \\
\text { alguna. La implementación abarcó a } \\
\text { seis países en un período de dos años. }\end{array}$ & $\begin{array}{l}\text { Tres operaciones adquiridas y la Oficina } \\
\text { Corporativa. La implementación abarcó a } \\
\text { cuatro países en total, en un período de dos } \\
\text { años. }\end{array}$ \\
\hline
\end{tabular}

\section{ANÁLISIS DE RESULTADOS}

\subsection{CONTRASTACIÓN DE LOS ENFOQUES PARA LA FUNDAMENTACIÓN DEL MODELO HIBRIDO}

En la Tabla 5 se describe la relación entre las técnicas y herramientas de gestión del cambio organizacional y los criterios RENGIS de innovación social, lo que permitió hacer una valoración cualitativa, con base en una escala descriptiva de tres niveles (Bajo, Medio, Alto), de acuerdo con la convergencia de los criterios RENGIS en procesos de gestión del cambio a lo interno de la organización. En la Tabla 6 se presenta el resumen de la valoración.

Se contrastó el proceso de gestión del cambio de la metodología MGSIC, con las fases del enfoque de innovación social, resultando en un alto grado de convergencia, por coincidir en aspectos relacionados con la transformación: desde la dimensión humana, el fortalecimiento de

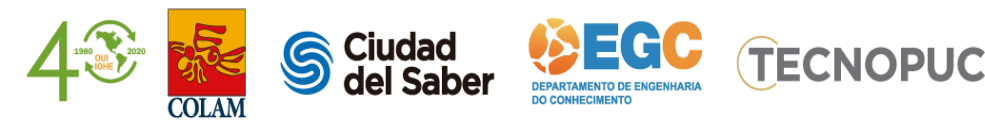


liderazgos organizacionales o comunitarios, hasta la sostenibilidad de soluciones que agregan valor y atiendan problemas complejos. Ello se traduce en beneficios de desarrollo, prosperidad y mejora de la calidad de vida, a un número plural de grupos de interés de la sociedad.

Tabla 3: Respuestas a las preguntas de Investigación - Caso 1

\begin{tabular}{|c|c|}
\hline PREGUNTAS & RESPUESTAS \\
\hline $\begin{array}{l}\text { ¿Qué cambio se requería } \\
\text { implementar y con qué } \\
\text { estructura y recursos se } \\
\text { contaba? }\end{array}$ & $\begin{array}{l}\text { Implementación de una herramienta tecnológica (ERP) y un modelo operativo } \\
\text { integral que garantizara mayor control y eficiencia en la gestión del negocio } \\
\text { y su desempeño en el nuevo ciclo de crecimiento de la empresa. Se gestionó } \\
\text { bajo la estructura de proyecto, con un presupuesto asignado. }\end{array}$ \\
\hline $\begin{array}{l}\text { ¿Cómo se abordó la } \\
\text { dimensión humana del } \\
\text { cambio para generar } \\
\text { compromiso a lo largo del } \\
\text { proyecto? }\end{array}$ & $\begin{array}{l}\text { Sesiones de sensibilización; Estrategia de Comunicación; Acciones de } \\
\text { Soporte y Acompañamiento (Coaching) y Alineación Organizacional } \\
\text { (Sistema de Reconocimiento e Incentivos). }\end{array}$ \\
\hline $\begin{array}{l}\text { ¿Cómo se garantizó el } \\
\text { éxito del cambio? }\end{array}$ & $\begin{array}{l}\text { Con el compromiso explícito de los Directivos y Líderes (Acuerdos formales); } \\
\text { Alineación Organizacional (Estructura con roles y responsabilidades y } \\
\text { capacitación según cargos); participación directa de los equipos de proyecto } \\
\text { en el diseño de la solución y su despliegue, y con la aplicación de herramientas } \\
\text { para la Gestión del Riesgo (Matriz de Impacto y Plan de Mitigación). }\end{array}$ \\
\hline $\begin{array}{l}\text { ¿Cómo se midió el éxito } \\
\text { del cambio? }\end{array}$ & $\begin{array}{l}\text { Evaluaciones por fase de cada proyecto (cumplimiento de hitos de las fases y } \\
\text { del compromiso organizacional); Sesiones de Lecciones Aprendidas; } \\
\text { Indicadores Integrales (cumplimiento de los tiempos y calidad de los } \\
\text { entregables del proyecto, auditoría post-implementación del cambio, \% de } \\
\text { ahorros por eficiencias, así como el retorno sobre la inversión). }\end{array}$ \\
\hline $\begin{array}{l}\text { ¿Cómo se gestiona la } \\
\text { Sostenibilidad? }\end{array}$ & $\begin{array}{l}\text { Implementación de Estrategia de Seguimiento y Medición del Cumplimiento } \\
\text { de los procesos, el mantenimiento de las estructuras organizacionales, el } \\
\text { conocimiento del modelo por parte de todo nuevo participante en el modelo. } \\
\text { Herramientas utilizadas: Formato de Autoevaluación por parte de cada } \\
\text { responsable de procesos del modelo, según nivel de cumplimiento de los } \\
\text { estándares establecidos. }\end{array}$ \\
\hline
\end{tabular}


Tabla 4 - Respuestas a las preguntas de investigación - Caso 2

\begin{tabular}{|c|c|}
\hline PREGUNTAS & RESPUESTAS \\
\hline $\begin{array}{l}\text { ¿Qué cambio se } \\
\text { requería implementar y } \\
\text { con qué estructura y } \\
\text { recursos se contaba? }\end{array}$ & $\begin{array}{l}\text { Unificación de las bases del modelo operativo de la Oficina Corporativa y } \\
\text { alineación de las operaciones recién adquiridas, con el mismo. Aumento de la } \\
\text { eficiencia a partir de ahorros por la fusión, bajo una misma estrategia de negocios } \\
\text { (financiera, cliente y mercado, procesos y estructura). De igual forma, la } \\
\text { constitución de una sola cultura organizacional. } \\
\text { Enfoque: inclusión en el ciclo de Planificación Estratégica bajo la modalidad de } \\
\text { proyecto, considerando los niveles ejecutivos de la organización y áreas de soporte } \\
\text { de las unidades de Estrategia e Innovación, TI, Comercial y RRH al } 25 \%-50 \% \\
\text { del tiempo. Presupuesto: a dos años, gestionado por el líder del proyecto. }\end{array}$ \\
\hline $\begin{array}{l}\text { ¿Cómo se abordó la } \\
\text { dimensión humana del } \\
\text { cambio para generar } \\
\text { compromiso a lo largo } \\
\text { del proyecto? }\end{array}$ & $\begin{array}{l}\text { Resumen del Método: Sesiones de sensibilización; generación de la visión } \\
\text { compartida del éxito del proyecto (se acordaron los indicadores de éxito); } \\
\text { Alineación Organizacional: diseño de una Estrategia Integral de Cambio enfocada } \\
\text { en un análisis de convergencias/divergencias en culturas; Estrategia de Desarrollo } \\
\text { de Líderes. }\end{array}$ \\
\hline $\begin{array}{l}\text { ¿Cómo se garantizó el } \\
\text { éxito del cambio? }\end{array}$ & $\begin{array}{l}\text { Resumen del Método: Alineación Organizacional a)Plan Estratégico: el proyecto } \\
\text { se incorporó a las iniciativas del plan a dos años, b) Estructura Organizacional: } \\
\text { Estrategia de Capacitación para los nuevos requerimientos de habilidades y } \\
\text { conocimientos por rol. c) Herramientas para la Gestión del Riesgo (Matriz de } \\
\text { Impacto y Plan de Mitigación). }\end{array}$ \\
\hline $\begin{array}{l}\text { ¿Cómo se midió el éxito } \\
\text { del cambio? }\end{array}$ & $\begin{array}{l}\text { Evaluación: avance de la iniciativa y logros de los hitos de fases del proyecto; } \\
\text { Ingresos, utilidad neta, adquisición de clientes; Acuerdos de Niveles de Servicio } \\
\text { (SLA) entre la Oficina Corporativa-Países. }\end{array}$ \\
\hline $\begin{array}{l}\text { ¿Cómo se gestiona la } \\
\text { Sostenibilidad? }\end{array}$ & $\begin{array}{l}\text { Enfoque: Alineación Organizacional: Gestión del Desempeño y Bonificación e } \\
\text { Incentivos Anuales para los equipos directivos y gerenciales. } \\
\text { Mecanismos de Gestión: evaluación del avance y la calidad de la implementación } \\
\text { de proyecto por país. } \\
\text { Mediciones post-implementación: auditorías del modelo operativo se incluye la } \\
\text { evaluación del marco de control interno (Políticas, Regulaciones y Normas). }\end{array}$ \\
\hline
\end{tabular}


Tabla 5: Análisis Comparativo de los enfoques basado en los criterios RENGIS

\begin{tabular}{|c|c|c|c|}
\hline \multicolumn{2}{|c|}{$\begin{array}{c}\text { Enfoque de Innovación Social de los } \\
\text { proyectos LabIS-UTP }\end{array}$} & \multicolumn{2}{|c|}{$\begin{array}{c}\text { Enfoque de Gestión del Cambio Organizacional } \\
\text { Metodología MGSIC }\end{array}$} \\
\hline $\begin{array}{l}\text { Fase y criterios } \\
\text { RENGIS }\end{array}$ & Descripción de la Fase & $\begin{array}{c}\text { Fase - Valoración de } \\
\text { criterios } \\
\end{array}$ & Descripción de la Fase \\
\hline $\begin{array}{l}\text { Criterios: C1-C5 - } \\
\text { C8 } \\
\text {-Sostenibilidad } \\
\text {-Iniciativa Genuina } \\
\text {-Desafío o Necesidad } \\
\text { Vigente }\end{array}$ & $\begin{array}{l}\text { Trabajo previo - Línea } \\
\text { Base e identificación de } \\
\text { problemática con técnicas } \\
\text { como Diálogo de Saberes. } \\
\text { (participación ciudadana), } \\
\text { para propiciar el } \\
\text { empoderamiento en la } \\
\text { comunidad. } \\
\text { Con la ayuda de un } \\
\text { experto se identifican los } \\
\text { contextos a abordar } \\
\text { dependiendo de la } \\
\text { problemática central } \\
\text { (Análisis Policontextural) } \\
\text { Realización de Talleres de } \\
\text { Preparación con } \\
\text { Mediadores y Talleres de } \\
\text { Inducción con líderes } \\
\text { comunitarios y otros } \\
\text { actores claves para } \\
\text { generar apropiación y } \\
\text { propiciar sostenibilidad }\end{array}$ & $\begin{array}{l}\text { FASE } \\
\text { SENSIBILIZACIÓN } \\
\text { Criterios: C1-C5-C8 } \\
\text {-Sostenibilidad (M) } \\
\text {-Iniciativa Genuina (A) } \\
\text {-Desafío o Necesidad } \\
\text { Vigente (A) } \\
\text { NIVEL DE } \\
\text { COINCIDENCIA CON } \\
\text { LOS CRITERIOS } \\
\text { RENGIS REQUERIDOS } \\
\text { EN LA FASE: MEDIO- } \\
\text { ALTO } \\
\text { Se trabaja con el personal } \\
\text { involucrado en el cambio } \\
\text { desde el inicio del } \\
\text { proceso }\end{array}$ & $\begin{array}{l}\text { Trabajo previo de preparación- } \\
\text { diagnóstico de la situación actual } \\
\text { Sesiones con el equipo directivo para } \\
\text { construir visión compartida, alcance, } \\
\text { resultados en términos cualitativos y } \\
\text { cuantitativos desde la perspectiva de } \\
\text { mayores beneficios para el cliente, el } \\
\text { negocio y riesgos. } \\
\text { Definición del equipo del proyecto de } \\
\text { cambio o transformación y de los } \\
\text { grupos de interés a ser impactados. } \\
\text { Esto es apoyado con un plan inicial de } \\
\text { espacios de comunicación para } \\
\text { generar el compromiso y los niveles } \\
\text { de alistamiento organizacional. }\end{array}$ \\
\hline $\begin{array}{l}\text { Criterios:C1-C2-C3- } \\
\text { C4-C5-C8 } \\
\text {-Sostenibilidad } \\
\text {-Impacto social } \\
\text {-Capacidad } \\
\text { Transformadora } \\
\text {-Originalidad } \\
\text {-Iniciativa Genuina } \\
\text {-Desafío o Necesidad } \\
\text { Vigente }\end{array}$ & 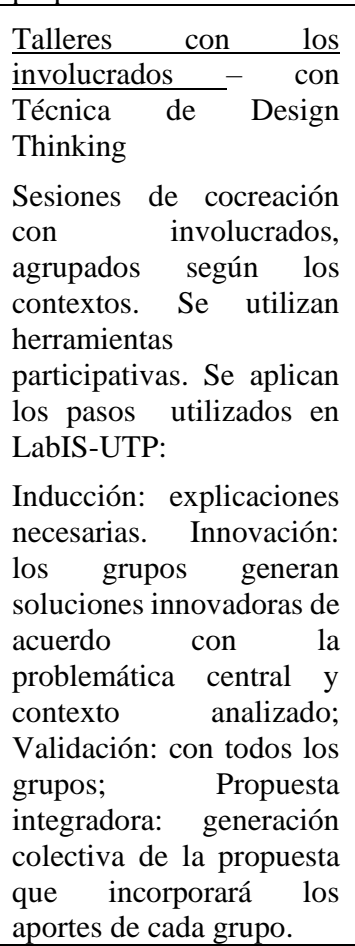 & $\begin{array}{l}\frac{\text { FASE }}{\text { DISEÑO Y }} \\
\frac{\text { DESARROLLO }}{\text { Criterios: C1-C3-C4-C5- }} \\
\text { C8 } \\
\text {-Sostenibilidad (A) } \\
\text {-Capacidad } \\
\text { Transformadora (A) } \\
\text {-Originalidad (M) } \\
\text {-Iniciativa Genuina (A) } \\
\text {-Desafío o Necesidad } \\
\text { Vigente (A) }\end{array}$ & $\begin{array}{l}\text { Sesiones conjuntas para: } \\
\text { Definición concreta del cambio a } \\
\text { implementar (líderes funcionales del } \\
\text { proyecto). } \\
\text { Diseño del nuevo modelo, sus } \\
\text { procesos, nuevas funcionalidades, las } \\
\text { configuraciones y pruebas de la } \\
\text { herramienta, para adaptarlas a la } \\
\text { realidad del negocio (líderes } \\
\text { funcionales). } \\
\text { Diseño de Estructura Organizacional } \\
\text { (Equipo de Gestión de Cambio). } \\
\text { Talleres de: } \\
\text { Lecciones Aprendidas en cada fase. } \\
\text { Dimensionamiento de Impactos } \\
\text { esperados en los procesos, sistemas, } \\
\text { roles y nuevos conocimientos } \\
\text { requeridos. del de de Mitigación de } \\
\text { Generación del Plan de } \\
\text { impactos. } \\
\text { Diseño final de Estrategia del } \\
\text { Cambio y plan detallado de } \\
\text { capacitación, de comunicación, y de } \\
\text { alineación organizacional, así como } \\
\text { de intervenciones de Coaching. }\end{array}$ \\
\hline
\end{tabular}


Tabla 5: Análisis Comparativo de las Fases de los enfoques en estudio basado en los criterios de innovación social RENGIS (Continuación)

\begin{tabular}{|c|c|c|c|}
\hline \multicolumn{2}{|c|}{$\begin{array}{c}\text { Enfoque de Innovación Social de los } \\
\text { proyectos LabIS-UTP }\end{array}$} & \multicolumn{2}{|c|}{$\begin{array}{c}\text { Enfoque de Gestión del Cambio Organizacional } \\
\text { Metodología MGSIC }\end{array}$} \\
\hline $\begin{array}{l}\text { Fase- Criterios } \\
\text { RENGIS }\end{array}$ & $\begin{array}{l}\text { Descripción de la } \\
\text { Fase } \\
\end{array}$ & $\begin{array}{c}\text { Fase- Valoración de } \\
\text { criterios } \\
\end{array}$ & Descripción de la Fase \\
\hline $\begin{array}{l}\text { Criterios: C1-C2- } \\
\text { C3-C4-C6 } \\
\text {-Sostenibilidad } \\
\text {-Impacto social } \\
\text {-Capacidad } \\
\text { Transformadora } \\
\text {-Originalidad } \\
\text {-Iniciativa } \\
\text { consolidada }\end{array}$ & $\begin{array}{l}\text { De acuerdo con la } \\
\text { propuesta } \\
\text { integradora se } \\
\text { genera un plan de } \\
\text { acción en detalle. } \\
\text { Se crean grupos } \\
\text { para el desarrollo } \\
\text { del trabajo } \\
\text { comunitario. } \\
\text { Se Preparan } \\
\text { técnicamente a los } \\
\text { líderes y } \\
\text { colaboradores. } \\
\text { Se Ejecuta el plan }\end{array}$ & $\begin{array}{l}\frac{\text { FASE }}{\text { ALINEACIÓN }} \\
\text { ORGANIZACIONAL } \\
\text { Criterios:C1-C3-C6-C8 } \\
\text { Sostenibilidad } \\
\text { Iniciativa Consolidada } \\
\text { Capacidad transformadora } \\
\text { NIVEL DE COINCIDENCIA } \\
\text { CON CRITERIOS RENGIS } \\
\text { REQUERIDOS: MEDIO }\end{array}$ & $\begin{array}{l}\text { Alineación de los elementos del } \\
\text { modelo operativo: desde la estrategia, } \\
\text { sus sistemas de gestión (incluyendo los } \\
\text { relacionados al talento), procesos de la } \\
\text { cadena de valor, la estructura } \\
\text { organizacional, sus herramientas, de } \\
\text { donde emerge la cultura organizacional. } \\
\text { Se concretan el Plan de Mitigación. } \\
\underline{\text { Se refuerzan las acciones de }} \\
\text { comunicación } \\
\underline{\text { Se ejecutan las primeras acciones de }} \\
\text { capacitación a nivel global, sobre la } \\
\text { inducción a las nuevas formas de } \\
\text { operar, enfocados en la parte humana } \\
\text { del cambio y liderazgo } \\
\text { Capacitación- conocimientos técnicos }\end{array}$ \\
\hline & $\begin{array}{l}\text { con la intervención } \\
\text { de los grupos de } \\
\text { trabajo y los } \\
\text { especialistas. }\end{array}$ & $\begin{array}{l}\text { FASE } \\
\text { EJECUCIÓN } \\
\text { Criterios: C1-C3-C6-C8 } \\
\text { Sostenibilidad } \\
\text { Capacidad transformadora } \\
\text { Iniciativa Consolidada } \\
\text { Repetitividad } \\
\text { NIVEL DE COINCIDENCIA } \\
\text { CON CRITERIOS RENGIS } \\
\text { REQUERIDOS: ALTO }\end{array}$ & $\begin{array}{l}\text { Despliegue del Plan de Cambio: desde } \\
\text { las acciones de Comunicación sobre el } \\
\text { lanzamiento y salida en productivo de } \\
\text { la nueva herramienta, hasta el } \\
\text { acompañamiento a los líderes en las } \\
\text { comunicaciones e impactos en sus } \\
\text { áreas. } \\
\text { Se propicia el compromiso } \\
\text { organizacional. } \\
\text { Se realiza el seguimiento de incidencias } \\
\text { por fallas de conocimiento y se realiza } \\
\text { capacitación adicional. }\end{array}$ \\
\hline $\begin{array}{l}\text { No se considera la } \\
\text { sostenibilidad } \\
\text { como fase, ya que } \\
\text { se aborda en la } \\
\text { cocreación a nivel } \\
\text { de diseño y en la } \\
\text { fase Transversal } \\
\text { del Feeback }\end{array}$ & & $\begin{array}{l}\text { FASE } \\
\text { SOSTENIBILIDAD } \\
\text { Criterios:C1-C3-C5-C6-C7-C8 } \\
\text { Sostenibilidad } \\
\text { Capacidad Transformadora }\} \\
\text { Iniciativa genuina } \\
\text { Iniciativa Consolidada } \\
\text { Repetitividad } \\
\text { Desafío Vigente } \\
\text { NIVEL DE COINCIDENCIA } \\
\text { CON CRITERIOS RENGIS } \\
\text { REQUERIDOS: ALTO } \\
\end{array}$ & $\begin{array}{l}\text { Se afianzan los nuevos conocimientos } \\
\text { y la adopción de la herramienta o } \\
\text { modelo operativo, con acciones de } \\
\text { seguimiento. } \\
\text { Se establecen mecanismos de } \\
\text { evaluación y de control a fin de } \\
\text { preservar los procesos, las estructuras, } \\
\text { la calidad y cumplimiento de los } \\
\text { objetivos del proyecto } \\
\text { Se propician periódicamente espacios } \\
\text { para compartir el conocimiento y } \\
\text { lecciones aprendidas. } \\
\text { Se incluyen las acciones de mejora. }\end{array}$ \\
\hline $\begin{array}{l}\text { FASE } \\
\text { Transversal }\end{array}$ & $\begin{array}{l}\text { Talleres lecciones } \\
\text { aprendidas y } \\
\text { Sistematización de } \\
\text { experiencias. } \\
\text { Generación de } \\
\text { nuevas ideas }\end{array}$ & $\begin{array}{l}\text { Acciones de Soporte a las fases } \\
\text { de 1-5 } \\
\text { Comunicación, Coaching y } \\
\text { Capacitación } \\
\text { NIVEL DE COINCIDENCIA } \\
\text { CON CRITERIOS RENGIS } \\
\text { REQUERIDOS: MEDIO }\end{array}$ & $\begin{array}{l}\text { Se realizan acciones transversales que } \\
\text { acompañan la gestión del equipo del } \\
\text { proyecto a lo largo de su desarrollo. } \\
\text { Se realizan acciones adicionales, según } \\
\text { se requiera con distintos equipos o áreas } \\
\text { de la organización de quienes depende } \\
\text { en gran medida el éxito del proyecto. }\end{array}$ \\
\hline
\end{tabular}


TABLA 6. Resumen de la valoración de criterios RENGIS vs. Gestión del Cambio

GRADO DE INTEGRACIÓN DE LA METODOLOGÍA MGSIC CON EL ENFOQUE DE INNOVACIÓN SOCIAL

\begin{tabular}{|c|c|c|c|}
\hline & BAJO & MEDIO & ALTO \\
\hline 1. SENSIBILIZACIÓN & & & \\
\hline
\end{tabular}

2. DISEÑO Y DESARROLLO

3. ALINEACIÓN

ORGANIZACIONAL

4. EJECUCIÓN

5. SOSTENIBILIDAD

6. SOPORTE TRANSVERSAL

BAJO: Se coincide en 35\% o menos $\quad$ MEDIO: Se coincide entre el 36\% - 69\% $\quad$ ALTO : Se coincide entre el 70\%-100\%

Tras la evaluación y análisis comparativo realizado se identifican las siguientes características deseables en el modelo híbrido: a) sistémico: fases interrelacionadas entre sí, que puedan ser realimentadas en cualquier estado del proyecto, mediante flujos de comunicación no lineal; b) que propicie la participación de grupos de interés en la identificación de problemáticas y soluciones innovadoras, mediante procesos de cocreación y de coejecución; c) centrado en procesos de transformación social y organizacional de base tecnológica; d) propiciador del auto aprendizaje en los grupos beneficiados y; e) formador de habilidades para la vida (resiliencia, liderazgo, aprendizaje autónomo, entre otros).

Se comprueba cómo los dos enfoques, por sus características centradas en procesos de transformación, pueden complementarse para desarrollar proyectos de cambio social por contener aspectos comunes tanto para abordar en una organización (privada, pública o no gubernamental), como en un conjunto de personas de un territorio o comunidad. Surge entonces la oportunidad de integrarlos en un modelo híbrido, que fortalezca las fases de Sensibilización, Alineación y Soporte Transversal, principalmente, con sesiones continuas de formación y desarrollo de habilidades para la vida, apoyados en sesiones de coaching con los diferentes grupos sociales, y en las distintas etapas del proyecto de cambio y transformación.

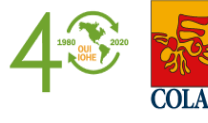


En adición, en la coyuntura actual, el modelo a generar podrá ser validado y aplicado en distintos grupos sociales en el tema de la COVID-19, con el fin de examinar los efectos producidos por la pandemia: desempleo, deterioro de la salud física y mental, crisis familiar, entre otros.

Esta experiencia investigativa, en su fase inicial, entre la Universidad Tecnológica de Panamá y el sector empresarial panameño en temas de transformación social y transformación organizacional, desde el punto de vista de la innovación social, abre todo un campo de investigación basado en la generación de nuevos modelos que incorporen mutuamente nuevas prácticas y conocimientos para abordar la complejidad actual de los sistemas sociales, con una mayor sinergia en el ecosistema de ciencia, tecnología e innovación panameño.

\section{AGRADECIMIENTOS}

A los investigadores del Centro de Investigación e Innovación Eléctrica, Mecánica y de la Industria de la Universidad Tecnológica de Panamá por su apoyo y participación en procesos de innovación social y a Inversiones Bahía, Ltd., corporación panameña de reconocida trayectoria, por su disposición para compartir buenas prácticas sobre gestión del cambio.

\section{REFERENCIAS}

Briceño, G. (2011). Polycontextural Logic: New Resource for Transdisciplinary Research in Sociology. Journal of Sociology Study. USA. Print ISSN: 2159-5526. Volume: 6/2011.

Escalante, R y Miñano, J. H. (1982), Investigación, organización y desarrollo de la comunidad. Nueva Biblioteca Pedagógica (vol. 2, pp 288-305). Editorial Oasis, N. 18, ISBN: 968505200X, 9789685052009.

HENKA (2013). Consulting: Metodología de Gestión Estratégica del Cambio, Res. 088-0022013.

Kotter, J. P. Leading Change(1996) Boston: Harvard Business School Press.

Lewin, K. (2018). A critical review of the role of leadership and employee involvement in organizational change. Journal of Innovation \& Knowledge. Elsevier. https://www.researchgate.net/publication/263685472_Sharpening_the_Focus_of_Forc e_Field_Analysis.

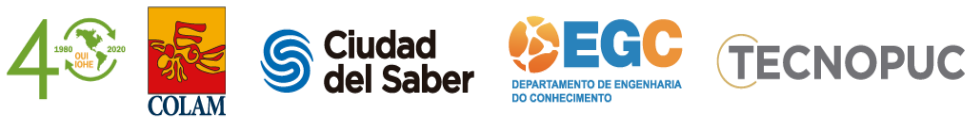


Mitleton-Kelly (2003). Sistemas complejos y perspectivas evolutivas sobre las organizaciones: la aplicación de la teoría de la complejidad a las organizaciones. ELSEVIER 2003, ISBN: 0-08-043957-8.

Moreno, S. y otros (2019). Codiseño de proyectos de innovación social: Caso del Distrito de Ñurum en Panamá, Proceding, pp 341-346. DOI=10.1109/IESTEC/46403.2019.00069.

McKinsey Global Survey results (2010). Encuesta Global, USA.

Roger, E. y otros (2003). Stage Theory of Organizational Change, University of Pennsylvania, USA. Health Behavior and Health Education.

Roger, E y otros (2007). The external environment's effect on management and strategy: A complexity theory approach.Management Decision 45(1) DOI: $10.1108 / 00251740710718935$.

Todnem, R. (2005). Organisational change management: A critical review, Journal of Change Management, 5:4, 369-380, DOI: $10.1080 / 14697010500359250$. http://dx.doi.org/10.1080/14697010500359250.

Turner, B. \& Croy, M. (2010). Waltzing with Da Vinci: the role of design thinking in project leadership. Paper presented at PMI® Global Congress 2010-North America, Washington, DC. Newtown Square, PA: Project Management Institute.

Vakola, M., \& Nikolaou, I. (2005). Attitudes towards organizational change. Employee relations.

Infografía

Agility Science Blog https://bvonderlinn.wordpress.com/2009/01/25/overview-of-ges-changeacceleration-process-cap/ Consultada: Agosto/2020

Center for Creative Leadership: https://www.ccl.org/articles/leading-effectively-articles/4types-of-coaching/ Coaching: Ejecutivo y de Equipos. Consultada Agosto/2020

Art of Hosting. https://www.artofhosting.org/.Consultada Agosto/2020 